\title{
Complex Method Mixed with PSO Applying to Optimization Design of Bridge Crane Girder
}

\author{
He Yan ${ }^{\mathrm{a}}$ and Wang Zongyan \\ ${ }^{1}$ School of Mechanical \& Power Engineering, North University of China, Shanxi, Taiyuan, China
}

\begin{abstract}
In engineer design, basic complex method has not enough global search ability for the nonlinear optimization problem, so it mixed with particle swarm optimization (PSO) has been presented in the paper,that is the optimal particle evaluated from fitness function of particle swarm displacement complex vertex in order to realize optimal principle of the largest complex central distance.This method is applied to optimization design problems of box girder of bridge crane with constraint conditions.At first a mathematical model of the girder optimization has been set up,in which box girder cross section area of bridge crane is taken as the objective function, and its four sizes parameters as design variables, girder mechanics performance, manufacturing process, border sizes and so on requirements as constraint conditions. Then complex method mixed with PSO is used to solve optimization design problem of cane box girder from constrained optimization studying approach, and its optimal results have achieved the goal of lightweight design and reducing the crane manufacturing cost . The method is reliable, practical and efficient by the practical engineer calculation and comparative analysis with basic complex method.
\end{abstract}

\section{Introduction}

Bridge crane is widely used in steel, metallurgy, chemical industry, building materials and other industries, because its forces in various working conditions are very complicated, the traditional design methods are often difficult to obtain accurate solutions. Designers have to use overlarge safety factor to ensure crane safety case, resulting the increase of steel material waste and construction cost[1]. Tian Deyu[2]using ANSYS optimization module ,Fan Xiaoning[3] using genetic algorithm, and Jin Chenguang[4] applying the chaos genetic algorithm for optimization design of crane girder, their optimization results than that of the traditional design are the weight reduction of girder. But the genetic algorithm usually uses binary coding, and also need to carry out complex replication, genetic, variation and other operations. Considering the optimization constraints of main girder in the optimization mathematical model including not only the requirements of strength, stiffness but also stability, so its optimization is a complex multidimensional optimization problem involving many constrains and nonlinear discrete variables. Many years ago, many researchers used the traditional constrained nonlinear algorithm, but there are shortcomings and limitations, such as such as large grid calculated amount and random direction method can only obtain the local optimal solution, Lagrange

\footnotetext{
acCorresponding author : Heyan@zbhy91@163.com
} 
multiplier method is suitable for optimization problem of solving equality constraints, and complex method needs the continuity and differentiability of objective function, so far the intelligent algorithm is the best way to solve this kind of problem.

Particle swarm optimization (PSO) is a global optimization algorithm simulating biological swarm intelligence theory. Particles usually carry on searching by guiding the optimization through individual particle information sharing and competition between groups, and by using iterative method to find the optimal solution. This method has more simple encoding rules than the genetic algorithm (GA), has shown its superiority in solving practical problems in the engineering field [5-6].

Aiming at optimization design problems of box girder of bridge crane with constraint conditions, the complex method mixed with PSO algorithm, in which the best particle evaluated from PSO will replace complex vertexes to obtain maximum central distance of complex. From the constraint processing technology compounding swarm intelligence algorithm, optimization design problem of bridge crane box girder section size will be solved and the direction of biological swarm intelligence applying to mechanical optimization design will be expanded.

\section{Complex method mixed with particle swarm optimization algorithm}

\subsection{Complex method}

Complex method is an important direct method for solving constrained optimization problems. The idea is to construct an initial complex shape with $\mathrm{K}$ vertices in the feasible region. The objective function values of each vertex of the composite shape are compared to find the vertex of maximum objective function (called the worst point), then according to certain rules find the practical new vertex which objective function value decline, and using this point instead of the dead, a new complex will be constituted, when its shape every changing once, it is moving a step to the most solution, until to the best approximation.

The calculation steps of the complex method are as follows [7]:

1) Select the vertex numbers $k$ of the complex shape, in general, $k$ is set as $n+1 \leq k \leq 2 n$, the initial complex consisted of $k$ vertexes is formed in the feasible region .

2) Calculate the objective function value of complex each vertex and compare them, then find the best point $\mathrm{X}_{\mathrm{L}}$, the worst point $\mathrm{X}_{\mathrm{H}}$ and the next to the worst point $\mathrm{X}_{\mathrm{G}}$.

3) Calculate the center $X_{C}$ of other $(k-1)$ vertex aside from the worst point $X_{H}$. Determine whether $\mathrm{X}_{\mathrm{C}}$ is feasible, if it is feasible point, then go to step 4);otherwise, determine the lower limit and the upper limit value of design variables, even let $\mathrm{a}=\mathrm{X}_{\mathrm{L}}, \mathrm{b}=\mathrm{X}_{\mathrm{H}}$, and then go to step 1), reconstructing initial complex.

4) Calculate the reflection point $X_{R}$ according to the equation (1), when necessary, change the value of the reflection coefficient until the reflection is successful, meeting the following equation (2). Then $\mathrm{X}_{\mathrm{R}}$ replaces $\mathrm{X}_{\mathrm{H}}$, and the new complex is constituted.

$$
X_{R}=X_{C}+\alpha\left(X_{c}-X_{H}\right)
$$

where, $\alpha$ is reflection coefficient, generally taking as equation (2).

$$
\alpha=1.3\left\{\begin{array}{c}
g_{j}\left(x_{R}\right) \leq 0 \\
f\left(x_{R}\right)<f\left(x_{H}\right)
\end{array} j=1,2, \mathrm{~L}, m\right)
$$

5) If the convergence condition which the root-mean-square of difference of the function value between each vertex and the good point is less than the error limit, namely equation (3) is satisfied, the calculation is terminated. Finally get the optimal solution, namely $X^{*}=X_{L}, f\left(X^{*}\right)=f\left(X_{L}\right)$. Otherwise, turn to step 2).

$$
\left\{\frac{1}{k-1} \sum_{j=1}^{k}\left[f\left(x_{j}\right)-f\left(x_{L}\right)\right]^{2}\right\}^{\frac{1}{2}} \leq \varepsilon
$$




\subsection{Particle swarm optimization algorithm}

In the PSO algorithm, each particle moves according to its velocity in the search space. The whole optimization process is that particle adjusts to its position and speed based on its optimal location and that of other individuals in its neighbour, then to accelerate moving toward global optimal value.

The standard particle swarm algorithm is shown in equation (4) (5):

$$
\begin{gathered}
v_{i d}(t+1)=\mathrm{w} v_{i d}(t)+c_{1} r_{1}\left(p_{i d}-x_{i d}(t)\right)+c_{2} r_{2}\left(p_{g d}-x_{i d}(t)\right) \\
x_{i d}(t+1)=x_{i d}(t)+v_{i d}(t+1)
\end{gathered}
$$

Where, $x_{i d}(\mathrm{t})$ is the current position of the particle $i, p_{i d}$ is the superior position of the particle $i$, which searches till now, $p_{g d}$ is the entire particle swarm's superior position. $c_{l}$ and $c_{2}$ are acceleration constants with positive values, usually set as $0 \sim 2 ; r_{l}$ and $r_{2}$ are random numbers in range[ 01 l]. $v_{i d}$ is the current flying velocity of the particle $i$ in range $\left[-v_{\max }, v_{\max }\right], v_{\max }$ is the maximum particle velocity, being non-negative number; $w$ is positive constant, called inertia; weight, which is linearly decreasing reducing with iteration, namely:

$$
w=w_{\max }-\frac{w_{\max }-w_{\min }}{T_{\max }} \mathrm{t}
$$

Where $w_{\max }$ and $w_{\min }$ are the maximum and minimum inertia weight values. Typically $w_{\max }=0.9 \sim 1.4, \quad w_{\min }=0.4 ; t$ is current iteration number, $T_{\max }$ is the maximum iteration number.

\subsection{The composite shape vertex is replaced with the best particle of PSO to achieve the maximum central distance}

At present, the reason resulting in the global search ability of complex method is that the domain surrounded by a new complex not large enough, which is randomly generated in a particular search domain or complex calculation process, Namely Hamming distances between the complex central points and each complex vertex are not large enough. Now the sum of Hamming distance is defined complex central distance represented as equation (7).

$$
\mathrm{D}_{c}=\sum_{i=1}^{\mathrm{k}} D_{i}
$$

If the optimal solution obtained by PS0 solution can replace a vertex of the complex to make the central distance of complex the largest, so that the search capability of the modified complex will be improved, and the size of the search has been expanded. The replacement criterion based on maximum complex central distance is as follow:

1) In PSO calculation, the initial population of each group of the complex shape method is corresponding to that of the PSO algorithm.

2) Replace $k$ vertexes of the current complex one by one with the better solution solved by the PSO, at the same time keeping the $(k-1)$ vertexes no changed to form $k$ new complex;

3) Calculate the central distance of $k$ new complexes, and set the complex having maximum central distance as that in next iteration.

\section{The establishment of optimization mathematical model of the main girder of bridge crane}

\subsection{Composition and working principle of the bridge crane}


The bridge crane is mainly composed of three parts, including car, bridge and electrical equipment. The three sets of motions constitute a bridge crane work three-dimensional space. Thus the bridge girder consisted of main beam and end beam is important component part of the bridge. Therefore, the main beam is the key point in the design of the crane, and its structure is a box type structure [8].

\subsection{Selection of design variables}

The optimization design goal of the crane girder is to reduce its weight on the basis of satisfying the performance of the whole machine. In the span being certain, the key factor affecting the weight of the main beam is its section area. It is mainly determined with the dimensions of upper and lower flange, main side webs and the reinforced rib plates whose weights are relate to the span[9,10]. Therefore, there are 4 design variables of main girder simplified in all, namely web height, flange width, web thickness, flange thickness, respectively, set as $\mathrm{x}_{1}, \mathrm{x}_{2}, \mathrm{x}_{3}, \mathrm{x}_{4}$ shown in Figure 1.

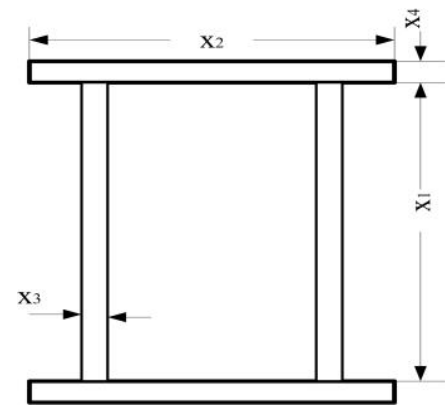

Figure 1. Box girder section of bridge crane

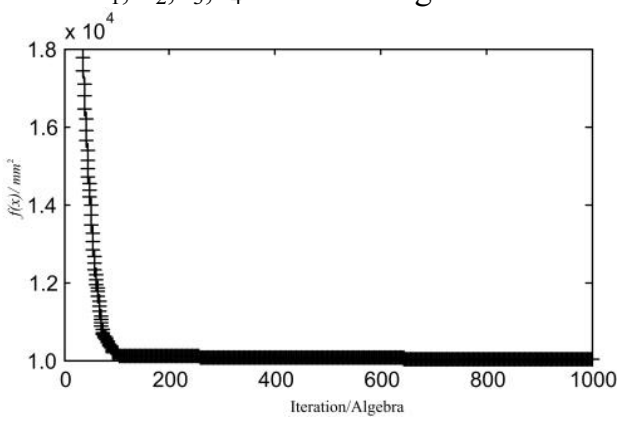

Figure2.Evolution curve of PSO algorithm

\subsection{Objective function}

When the main beam span and steel density are certain, the weight of the girder is proportional to its cross-sectional area, so the section area of the main beam is taken as the objective function. It is established for the quality of single girder in double girder bridge crane to be the lightest, which can be expressed as equation (8).

$$
f(x)=2\left(x_{1} x_{3}+x_{2} x_{4}\right)
$$

\subsection{Constraint conditions}

(1) Intensity constraint

Corner point stress of the flange in cross section is the largest, so the bending stress constraint can be represented as equation (9).

$$
\sigma=\frac{T_{V} x_{1}}{2 I_{x x}}+\frac{T_{H} x_{2}}{2 I_{y y}} \leq[\sigma]
$$

Where, $T_{v}$ denotes the sum of the bending moment in the vertical direction, which is caused by the fixed load and moving load on the beam section; $T_{\mathrm{H}}$ denotes the sum of the bending moment generated by the horizontal inertia load when the cart is braking; $I_{x x}, I_{y y}$ denote inertia moment of beam cross section to the $\mathrm{X}$ axis and the $\mathrm{Y}$ axis, respectively; $[\sigma]$ is allowable stress of main beam design.

$$
g_{1}(x)=\frac{3 L}{4}\left[\frac{P_{1}+\rho\left(x_{1} x_{3}+x_{2} x_{4}\right) L}{3 x_{1} x_{2} x_{4}+x_{1}^{2} x_{3}}+\frac{P_{2}}{3 x_{1} x_{2} x_{3}+x_{2}^{2} x_{4}}\right]-[\sigma] \leq 0
$$

Where, $\mathrm{P}_{1}$ and $\mathrm{P}_{2}$ denote the maximum vertical static load and the maximum horizontal dynamic load, respectively. $\rho$ is the density of main beam steel material, $L$ is span of the main beam. 
(2) Stiffness constraint

$$
\frac{P_{1} L^{3}}{48 E I_{x x}} \leq\left[f_{v}\right]
$$

Where $\mathrm{E}$ is the elasticity modulus of steel material, in general, $\left.\mathrm{E}=2.06 \times 105 \mathrm{MPa} ; f_{v}\right]$ is main girder allowable deflection, $\left[f_{v}\right]=\mathrm{L} / 700$;After finishing the simplification, the stiffness constraint is expressed as equation (12).

$$
g_{2}(x)=\frac{P_{1} L^{3}}{3 x_{1}^{2} x_{2} x_{4}+x_{1}^{3} x_{3} \times 9.9 \times 10^{6}}-\left[f_{V}\right] \leq 0
$$

(3) Process constraint

$$
\begin{aligned}
& g_{3}(x)=\frac{x_{2}}{x_{4}}-60 \leq 0 \\
& g_{4}(x)=\frac{x_{1}}{x_{3}}-160 \leq 0
\end{aligned}
$$

(4) Boundary constraint

$$
\begin{aligned}
& g_{5}(x)=5-x_{3} \leq 0 \\
& g_{6}(x)=5-x_{4} \leq 0
\end{aligned}
$$

\section{Realization of optimization design}

\subsection{Parameters Setting}

(1) Parameters setting in the particle swarm optimization algorithm

Set the various parameters of PSO algorithm, including particle numbers $m=20$, the inertia weight $w_{\max }=1.2, w_{\min }=0.4$, acceleration constants $c_{1}=c_{2}=2$, the maximum iterations number $T_{\max }=1000$, the maximum particle velocity $v_{\max }=1$, the fitness goal $\varepsilon=0.1$.

(2) The crane structure parameters setting

Taking the double girder crane in common use as an example, the basic parameters are set as follows:

$$
P_{1}=1.2 \times 10^{5} \mathrm{~N}, P_{2}=1.2 \times 10^{4} \mathrm{~N}, L=10.5 \mathrm{~m},[\sigma]=140 M P_{a},\left[f_{v}\right]=L / 700 \cdot x_{1} \in[660,900], x_{2} \in[240,400],
$$

$x_{3} \in[5,32], x_{4} \in[5,32]$, and four variables of each dimension are $\mathrm{mm}$ as the unit. Generally the variation intervals of web height and flange width of the main beam are set to an integer multiple of $5 \mathrm{~mm}$; when flange thickness and web thickness are less than $30 \mathrm{~mm}$, the variation intervals are set to $0.5 \mathrm{~mm}$; when they are more than $30 \mathrm{~mm}$, the change intervals are set to $1 \mathrm{~mm}[10]$.

\subsection{The Optimization Results}

The optimization problem involves one objective function and six constraints. Complex method mixed with PSO (CM-PSO) is programmed by MATLAB for optimization. The $f(x)$ is taken as fitness in PSO, and particle swarm evolutionary iteration process has been shown in Figure 2, the optimization results with the complex mixed with PSO method and simple complex method (CM) are compared .They are shown in Table 1.

\subsection{Results analysis}


The optimal results in Table 1 show that the area of the main beam section is $14819 \mathrm{~mm}^{2}$ by the complex method, and that is $11890 \mathrm{~mm}^{2}$ by complex method mixed with PSO, which has reduced $19.77 \%$. Seen from Figure 2, the PSO algorithm running in groups quickly converges to the optimal solution. After the optimal particle replacing complex vertex, enlarging the maximum complex central distance, and expanding the search space of feasible solutions, meanwhile increasing the diversity of population in optimal process, so the global convergence of algorithm has greatly improved.

Table 1.Optimization results of main girder structure size

\begin{tabular}{|l|c|c|}
\hline Mariable (unit) & CM & $\begin{array}{c}\text { CM- } \\
\text { PSO }\end{array}$ \\
\hline web height $\mathrm{x}_{1} \quad(\mathrm{~mm})$ & 784 & 775 \\
\hline flange width $\mathrm{x}_{2} \quad(\mathrm{~mm})$ & 356 & 345 \\
\hline web thickness $\mathrm{x}_{3} \quad(\mathrm{~mm})$ & 6 & 5 \\
\hline flange thickness $\mathrm{x}_{4}(\mathrm{~mm})$ & 7.6 & 6 \\
\hline Section area $\mathrm{f}(\mathrm{x})\left(\mathrm{mm}^{2}\right)$ & 14819 & 11890 \\
\hline Iterative algebra $(\mathrm{g})$ & 690 & 270 \\
\hline Time $\quad(\mathrm{s})$ & 14 & 3 \\
\hline
\end{tabular}

\section{Conclusions}

In the complex method mixed with PSO, the optimal particle in PSO replaces complex vertex to realize the maximum complex central distance. Starting from method of constraint handling techniques and swarm intelligence, the problems of optimization design are solved, which improves this modified complex search ability, and makes the search scale enlargement, so that the optimal solution speed and accuracy are enhanced. Through new algorithm the girder cross-sectional sizes of the bridge crane are optimized, the section area of the main beam has greatly decreased, and we achieve the goal of both the compact lightweight structure and mechanical properties. This method can be applied to the similar operating mechanism.

\section{References}

1. L. Riqi. Analysis and Optimization Research on the structure of box girder of overhead traveling crane[D].Harbin: Northeast Forestry University, 4 (2012)

2. T. Deyu, S. Dawen, S. Tingting etc. Optimization design of main girder of bridge crane [J]. New Technology \& New Process, 11 (2013)

3. F. Xiaoning, C. Hui, X. Gening. Optimization design software for overhead traveling crane based on annealing genetic algorithm [J]. Hoisting and conveying machinery, 5 (2013)

4. J. Chenguang. Optimization Design of Bridge Crane Box Girder Based on Chaotic Genetic Algorithm [D].North University of China. 6 (2014)

5. H. Yourui. Intelligent optimization algorithm and its application [M]. Beijing: National Defense Industry Press, 1 (2008)

6. Y. Yafei. The Method of Particle Swarm Optimization Solving Constrained Problems [D]. Beijing: China University of Geosciences, (2012)

7. L. Zhifeng. Mechanical optimization design [M]. Beijing: Higher Education Press, 2011.3

8. X. Gening. The metal structure of mechanical equipment[M].Beijing: Mechanical Industry Press, (2009)

9. T. Fang, S. Juan, Z. Yu. Technique of constrain processing based on punishment and reparation strategy [J]. Computer engineering \& design, 12(2006)

10. Y. Qinglin, T. Yuanfang, S. Nanxi etc. Particle swarm optimization algorithm combined with penalty function is used to optimize the main beam of bridge crane [J]. Hoisting and conveying machinery, 4(2015) 